Urinary phytoestrogen levels and frailty in older American women and the National Health and Nutrition Examination Survey (NHANES) 1999-2002: a cross-sectional study

Eichholzer, Monika ; Richard, Aline ; Walser-Domjan, Esther ; Linseisen, Jakob ; Rohrmann, Sabine

DOI: https://doi.org/10.1159/000356453

Posted at the Zurich Open Repository and Archive, University of Zurich

ZORA URL: https://doi.org/10.5167/uzh-86429

Journal Article

Published Version

Originally published at:

Eichholzer, Monika; Richard, Aline; Walser-Domjan, Esther; Linseisen, Jakob; Rohrmann, Sabine (2013). Urinary phytoestrogen levels and frailty in older American women and the National Health and Nutrition Examination Survey (NHANES) 1999-2002: a cross-sectional study. Annals of Nutrition Metabolism, 63:269-276.

DOI: https://doi.org/10.1159/000356453 


\title{
Urinary Phytoestrogen Levels and Frailty in Older American Women of the National Health and Nutrition Examination Survey (NHANES) 1999-2002: A Cross-Sectional Study
}

\author{
Monika Eichholzer ${ }^{a} \quad$ Aline Richard $^{\mathrm{a}}$ Esther Walser-Domjan ${ }^{\mathrm{a}} \quad$ Jakob Linseisen $^{\mathrm{b}}$ \\ Sabine Rohrmann ${ }^{\mathrm{a}}$ \\ ${ }^{a}$ Division of Cancer Epidemiology and Prevention, Institute of Social and Preventive Medicine, University of Zurich,

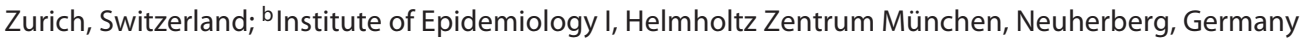

\section{Key Words}

Frailty · Phytoestrogens - National Health and Nutrition

Examination Survey · Elderly women

\begin{abstract}
Background/Aims: A deficit of various hormones during the process of aging and/or a heightened inflammatory state may be causally linked to the development of frailty. Phytoestrogens as weak estrogens, antioxidants, and anti-inflammatory agents may counteract this process. Methods: In a cross-sectional study including two cycles of the National Health and Nutrition Examination Survey (NHANES, i.e. 1999-2002), logistic regression was used to analyze the association between urinary concentrations of isoflavones and lignans and frailty in 600 females aged 50 years or older (median age 66.5 years). Participants were classified as 'frail' (meeting 3 or more of the 5 frailty criteria), 'prefrail' (meeting 1 or 2 of the criteria), or 'robust' (meeting none of the criteria). Four percent were frail. $\boldsymbol{R e}$ sults: For all of the phytoestrogens considered, the unadjusted OR were lower than 1 but generally not statistically significant aside from the association with O-desmethylan-
\end{abstract}

\section{KARGER}

E-Mail karger@karger.com

www.karger.com/anm golensin (O-DMA) (OR $=0.76 ; 95 \% \mathrm{Cl} 0.61-0.92)$. Multivariate analysis did not attenuate this finding $(\mathrm{OR}=0.74 ; 95 \%$ $\mathrm{Cl}$ 0.61-0.90). Conclusions: This first analysis of the relationship between phytoestrogens and frailty revealed an inverse association between urinary O-DMA levels and frailty in women. However, the number of frail women was low. Although this finding may be confounded or biased, it seems worthwhile to intensify research on the potential preventive effects of O-DMA.

๑) 2013 S. Karger AG, Basel

\section{Introduction}

Frailty is characterized by a decreased resistance to stress due to cumulative declines in multiple interrelated physiologic systems $[1,2]$. This results in an increased risk of falls, disability, hospitalization, institutionalization, and mortality [2]. Frailty is the consequence of impairment of multiple subsystems, such as sarcopenia [3], nutritional deficiencies [4], hormonal declines [5], or inflammation [6], and knowledge of the pathophysiological mechanisms of frailty remains limited. 
The most widely used definition of frailty is the one by Fried et al. [2], which includes unintentional weight loss, self-reported exhaustion, poor grip strength, slow walking speed, or low physical activity. Frailty is rarely observed in the absence of (subclinical) chronic disease. Unintentional weight loss may be the consequence of catabolic processes in the course of an underlying disease or it may be related to low energy and nutrient intakes. Accordingly, the characteristics of 65- to 79-year-old women becoming frail during follow-up in the Women's Health Initiative study were: high and low body weight, smoking, depression, cardiovascular disease (CVD), diabetes, and chronic obstructive pulmonary disease [7]. Furthermore, in the InCHIANTI study [8] more than half of the frail participants had insufficient intakes of energy, protein, calcium, iron, zinc and the vitamins A, D, E, C, and/or folate. In this cross-sectional study a low energy intake was associated with exhaustion, and a low nutrient score of proteins and vitamins was associated with weakness.

However, frailty is not a unidirectional process [9]. It is possible to recover from frailty to prefrailty and nonfrailty conditions, respectively [10]. This makes the adoption of preventive measures attractive. Accordingly, trials investigating the effect of hormone replacement therapy (HRT) in women on muscle mass and strength suggest a direct relationship with levels of plasma estradiol, but their results remain conflicting [11]. There is even evidence that usual-dose estrogen may accelerate muscle loss [12] and that higher levels of endogenous estradiol are directly associated with frailty [3]. It is therefore of interest to examine the effect of natural, weak estrogens on the risk of frailty in elderly women, without the health risks associated with the use of HRT.

Phytoestrogens are naturally occurring plant constituents found in many human foodstuffs. As their name implies, phytoestrogens may act as weak estrogens [13]. In addition, they may act as antioxidants and anti-inflammatory agents [14]. There are three main groups of phytoestrogens, i.e. isoflavones (in soybeans and other legumes), lignans (in flaxseed and berries), and coumestans (in soybean sprouts) [13]. The major soy isoflavones are daidzin and genistin, the glycoside conjugates of daidzein and genistein [15]. After ingestion, daidzein can be metabolized by gut bacteria into diverse compounds such as equol and $\mathrm{O}$-desmethylangolensin $(\mathrm{O}$ DMA). About $30-35 \%$ of the Western population and up to $60 \%$ of vegetarians and Asians possess the ability to produce equol, but these percentages vary between studies, depending, among other things, on the urine equol concentration used as the cutoff and the equol intake via milk products [16-19]. Equol is 100 times more estrogenic than daidzein [20]. Several animal products such as cheese, eggs, and milk also contain equol [18, 21]. The major human lignans are enterolactone and enterodiol, formed from plant precursors by the gut microflora [13].

So far, the health effects of phytoestrogens have often been studied by measuring the dietary intake of these compounds by study participants. However, measurement of the concentration of phytoestrogens in urine takes into account interindividual variations in microbial synthesis [22] and is independent of errors due to imprecise dietary assessments and databases. To determine whether frailty, based on a modified index of Fried et al. [2; see also 23], is associated with urinary phytoestrogen concentrations of isoflavones, lignans, genistein, daidzein, equol, equol of equol producers, $\mathrm{O}$ DMA, enterolactone, and enterodiol, we used the data of women aged 50 years or older from the National Health and Nutrition Survey (NHANES) 1999-2002. To our knowledge, this is the first study to investigate the association between these urinary phytoestrogen levels and frailty.

\section{Methods}

\section{Study Design}

NHANES is an on-going cross-sectional study representative of the population of the USA, which is being conducted by the National Center for Health Statistics (NCHS). Data are released for 2 successive years (i.e. 1999/2000, 2001/2002, etc.). NHANES is a program of studies designed to assess the health and nutritional status of adults and children in the USA. The survey is unique in that it combines interviews and physical examinations.

The NHANES interview includes demographic, socioeconomic, dietary, and health-related questions. The examination component consists of medical, dental, and physiological measurements, as well as laboratory tests administered by highly trained medical personnel. All data are anonymized.

The sample for the survey is selected to represent US populations of all ages. A complex, multistage, probability sampling design is used to select participants representative of the civilian, noninstitutionalized US population. To produce reliable statistics, NHANES oversamples individuals aged 60 years or older, AfricanAmericans, and Hispanics.

Participants $(n=21,004)$ were available from the $1999 / 2000$ and 2001/2002 NHANES cycles. After exclusion of participants younger than 50 years, our sample consisted of 4,983 individuals. In a further step, all men were excluded, leaving 2,551 women in our data set. Of these women, 617 had available data on urinary phytoestrogen measurements. After defining frailty variables, finally 600 women were included in our analyses. 
The NHANES study protocols are in accordance with the guidelines put forth in the Declaration of Helsinki, and all procedures involving human subjects were approved by NCHS Research Ethics Review Board. Written informed consent was obtained from all subjects.

\section{Measurement of Urinary Phytoestrogen Concentration}

Urine specimens were processed, stored, and shipped to the Division of Environmental Health Laboratory Sciences, National Center for Environmental Health, Centers for Disease Control and Prevention, for analysis [24]. Vials were stored under appropriate frozen $\left(-20^{\circ} \mathrm{C}\right)$ conditions until shipment to the National Center for Environmental Health for testing. An HPLC-MS/MS method was used to determine urinary phytoestrogen concentrations in NHANES [12]. The methods were adapted, which resulted in improved selectivity, sensitivity, and precision for the quantitative detection of phytoestrogens in human urine and serum. The method uses enzymatic deconjugation of the phytoestrogens followed by solid-phase extraction and reverse-phase HPLC to resolve the analytes. The phytoestrogens are detected using a Sciex API III heated nebulizer-atmospheric pressure chemical ionization (HNAPCI) interface coupled with MS/MS. The following phytoestrogens were measured in both NHANES cycles (1999/2000 and 2001/2002): daidzein, genistein, equol, O-DMA, enterodiol, and enterolactone. In our analyses, isoflavones were represented by daidzein, genistein, equol, and O-DMA. Lignans consisted of enterodiol and enterolactone.

The creatinine concentration was analyzed using a Jaffé rate reaction with a CX3 analyzer (Beckmann Instruments, Brea, Calif., USA) [25]. In our analyses, we used creatinine to standardize urinary phytoestrogen concentrations. Phytoestrogen concentrations were expressed as micrograms per gram of creatinine.

\section{Assessment of Frailty}

We based our analyses on the frailty score developed by Fried et al. [2], which was modulated to the data available in NHANES III by Wilhelm-Leen et al. [23] and to the data available in NHANES 1999-2002. This adapted score comprised the following 5 items: (1) low body weight for height, defined as a BMI $\leq 18.5$; (2) slow walking, defined as the slowest quintile by sex in a timed 20 -ft walk; (3) weakness, defined as present if participants answered 'some difficulty', 'much difficulty', or 'unable to do' when asked how much difficulty they have 'lifting or carrying something as heavy as ten pounds (like a sack of potatoes or rice)'; (4) exhaustion, defined as present if participants answered 'some difficulty', 'much difficulty', or 'unable to do' when asked how much difficulty they have 'walking from one room to the other on the same level', and (5) low physical activity, defined as present if participants answered 'less active' when asked: 'Compared with most women your age, would you say that you are more active, less active or about the same?'

In our analysis we included participants with valid information on at least 3 of the 5 frailty symptoms. One item was missing for 60 women, and 2 items were missing for 115 women. In total, all of the participants with 1 or 2 components missing emerged as belonging to the subgroup 'robust' (see below).

Women meeting 3 or more of the 5 criteria were classified as 'frail', those with 1 or 2 criteria were classified as 'prefrail', and those meeting none of the criteria were classified as 'robust'. In addition, the frailty variable was dichotomized into 'frails' ( 3 or more frailty criteria) versus 'nonfrails' ( $0-2$ frailty criteria) for the purposes of our analyses [2, 23].

\section{Other Explanatory Variables}

Age, sex, race/ethnicity, poverty income ratio (PIR), cigarette smoking, and use of HRT were assessed using a questionnaire. Weight was measured to the nearest $0.01 \mathrm{~kg}$ using an electronic digital scale while the participant was wearing a paper shirt and paper pants and foam slippers. Height was measured to the nearest $0.1 \mathrm{~cm}$ using a stadiometer. The BMI was calculated as the weight in kilograms divided by the square of the height in meters. A selfreported history of congestive heart failure, coronary heart disease, heart attack, or stroke was used to define prevalent CVD, and a self-reported history of a diagnosis of cancer (other than nonmelanoma skin cancer) was used to define prevalent cancer. Participants were considered to be diabetic if they reported a history of a diagnosis of diabetes or were users of insulin or diabetic medication. A self-reported history of hypertension was assessed if the participants had been told at least twice by a doctor that they had hypertension and/or if the participants took medication.

\section{Statistical Analysis}

Analyses were performed using STATA version 11 (Stata Corporation, College Station, Tex., USA), and $\mathrm{p}<0.05$ was considered statistically significant (two-sided tests). Sampling weights were applied according NHANES guidelines to produce estimates that were representative of the noninstitutionalized, civilian US population [26].

We used medians to compare phytoestrogen levels in the categories of frailty (robust, prefrail, and frail) and percentages for demographic variables. Differences between groups were evaluated using a univariate $t$ statistic or $\chi^{2}$ test, respectively. All significance tests were two-sided using $\mathrm{p}<0.05$ as the level of statistical significance.

Frailty was dichotomized into frail and nonfrail (prefrail and robust) for logistic regression, which was used to calculate OR and 95\% CI. We did combine prefrails with robusts and not with frails because we were not able to fully distinguish between robusts and prefrails due to missing data. Creatinine-standardized phytoestrogen concentrations were continuous variables. Besides the unadjusted model, we adjusted in a first model for age $(\geq 50-59,60-69,70-79$, and $>80$; equal results were found when we considered age as a continuous variable) and race/ethnicity (non-Hispanic black, non-Hispanic white, Mexican-American, and other). With the multivariable model, we wanted to evaluate potential confounders that influence frailty. Thus, besides age and race/ethnicity, we also included the PIR [which is the proportion of the self-reported family income to the US censusbased poverty threshold value for each calendar year adjusted for inflation and the age of the family reference person (a PIR value of 1 or greater indicates an income above the poverty level, whereas a PIR value below 1 indicates poverty)], smoking history (former, current, or never), current HRT (yes or no), and chronic diseases, i.e. CVD, hypertension, diabetes, and cancer. Furthermore, we performed a subanalysis for equol concentration in equol producers. Equol producers were defined as having an equol concentration over the detection limit. Moreover, we conducted a sensitivity analysis comparing the prevalence of frailty symptoms and the distribution of frailty scores in the women of our sample $(n=600)$ with those in women aged 50 years or older not included in our sample $(n=1,951)$ to evaluate differences between these two groups of women. 


\section{Results}

Tables 1 and 2 summarize the biochemical, lifestyle, and clinical variables of the participants with data on urinary phytoestrogen concentrations and frailty. Of the 600 women (unweighted $\mathrm{n}$ ) included in the present analysis, $64.4 \%$ belonged to the category robust, $31.6 \%$ belonged to the category prefrail, and $4.0 \%$ belonged to the category frail. Comparing our sample to the nonincluded women in the same age range $(n=1,951), 70.1 \%$ belonged to the category robust, $27.1 \%$ belonged to the category prefrail, and $2.9 \%$ belonged to the category frail. The prevalence of frailty symptoms in the nonincluded sample was distributed as follows: weakness, $43.3 \%$; slow walking, $19.3 \%$; exhaustion, $18.3 \%$; low physical activity, $20.1 \%$, and low BMI, $1.6 \%$ (see table 1 for a comparison with the included women).

In relation to phytoestrogens, a higher percentage of frail women versus prefrail and robust women had a somehow lower urine concentration of lignans, equol, $\mathrm{O}$ DMA, enterodiol, and enterolactone. The opposite was true for isoflavones, daidzein, and genistein. Among all women, $63.6 \%$ were equol producers. The equol concentration in frail equol producers was slightly higher than in robust women.

Moreover, a higher percentage of frails versus prefrails/robusts were HRT users. A history of CVD, diabetes, and hypertension, but not of cancer, was more often noted among frail women than prefrail and robust women (for statistically significant differences, see the $\mathrm{p}$ values in table 2).

Table 3 presents the associations between phytoestrogen concentrations and frailty. Unadjusted OR were below 1 for all phytoestrogens considered, but neither was statistically significant, with the exception of O-DMA ( $\mathrm{OR}=0.76$; 95\% CI 0.61-0.92). Models adjusting for age and race/ethnicity revealed similar results, again with a significantly reduced risk of frailty with increasing $\mathrm{O}$ DMA urinary levels $(\mathrm{OR}=0.76$; $95 \%$ CI $0.62-0.92)$. The latter finding was not attenuated in multivariate analysis $(\mathrm{OR}=0.74 ; 95 \% \mathrm{CI} 0.61-0.90)$. Considering age as a continuous variable did not affect the results.

\section{Discussion}

To the best of our knowledge, this is the first study to investigate the association between urinary phytoestrogen concentrations and frailty. In our cross-sectional study, representative of noninstitutionalized US women aged 50
Table 1. Prevalence of frailty symptoms and distribution of frailty scores (unweighted $n=600$ ) in women aged 50 years or older from NHANES 1999-2002

\begin{tabular}{lrr}
\hline & $\mathrm{n}^{\mathrm{a}}$ & $\%^{\mathrm{b}}$ \\
\hline Frailty score [23] & & \\
0 & 371 & 64.4 \\
1 & 150 & 22.2 \\
2 & 57 & 9.4 \\
$3-5$ & 22 & 4.0 \\
Frailty score components & 175 & 33.7 \\
$\quad$ Weakness & 121 & 28.3 \\
Slow walking & 56 & 11.6 \\
Exhaustion & 99 & 16.1 \\
Low physical activity & 9 & 1.8 \\
Low BMI & & \\
\hline a Unweighted. ${ }^{\mathrm{b}}$ Weighted. & & \\
\hline
\end{tabular}

years or older, $64.4 \%$ were robust, $31.6 \%$ were prefrail, and $4.0 \%$ were frail. The low percentage of frails observed in the present analysis most probably reflects the rather low age group considered. Furthermore, some of the women may not yet have experienced menopause (no data on menopausal status were available), which is of importance for musculoskeletal health [27]. In addition, there may be an underestimation of the proportion of frails since NHANES requires participants to come in for examination. A considerable number of frails might have been physically unable to participate, and not all of the frail women might have used the alternative of a home examination. Accordingly, the prevalence of frailty in the community-dwelling population of Western countries has been estimated to be about $6-20 \%$ using various definitions of frailty $[28,29]$. In comparison, in the first study estimating the prevalence of frailty in Taiwan, $4.9 \%$ of seniors aged $65-103$ years were frail, $40 \%$ were prefrail, and $55.1 \%$ were nonfrail (no criterion of frailty) [28], i.e. the prevalence of frailty was lower than in Western countries. Moreover, we confirmed previous observations showing a higher risk of frailty for subjects with chronic disease such as diabetes [30], hypertension [31], or a history of CVD [32]. The urinary phytoestrogen concentrations in the present study were comparable to those in previous studies in the US population [20] but lower than, for example, those in people living in Japan or Vietnam [33].

We observed no significant association between frailty and urinary levels of isoflavones, lignans, genistein, enterodiol, enterolactone, daidzein, or equol in women. There was one exception: increasing urinary O-DMA lev- 
Table 2. Age-standardized baseline characteristics by frailty status in women aged 50 years or older from NHANES 1999-2002

\begin{tabular}{|c|c|c|c|c|c|c|c|}
\hline & \multicolumn{3}{|l|}{ Frail $^{\mathrm{a}}$} & \multicolumn{3}{|c|}{ Prefrail $^{\mathrm{b}}$ and robust ${ }^{\mathrm{c}}$} & \multirow[t]{2}{*}{$\mathrm{p}$ value } \\
\hline & median & Q1 & Q3 & median & Q1 & Q3 & \\
\hline \multicolumn{8}{|l|}{ Phytoestrogens, $\mu \mathrm{g} / \mathrm{g}$ creatinine } \\
\hline Isoflavones $^{\mathrm{d}}$ & 116.8 & 28.4 & 423.6 & 104.1 & 46.2 & 339.9 & 0.033 \\
\hline Lignans $^{\mathrm{e}}$ & 394.2 & 107.6 & 766.0 & 476.6 & 192.7 & $1,038.7$ & 0.145 \\
\hline Daidzein & 62.6 & 14.7 & 214.7 & 48.3 & 16.4 & 169.6 & 0.057 \\
\hline Equol & 5.0 & 2.4 & 11.8 & 7.8 & 3.7 & 17.3 & 0.024 \\
\hline Equol among equol producers & 14.1 & 6.0 & 38 & 11.9 & 6.7 & 24.1 & 0.046 \\
\hline Genistein & 46.2 & 11.7 & 115.8 & 25.0 & 10.0 & 89.6 & 0.081 \\
\hline O-DMA & 1.3 & 0.4 & 7.4 & 2.9 & 0.6 & 19.8 & 0.021 \\
\hline Enterodiol & 40.1 & 10.5 & 100.0 & 44.0 & 18.3 & 93.9 & 0.569 \\
\hline Enterolactone & 316.9 & 58.7 & 417.2 & 402.4 & 133.3 & 944.6 & 0.003 \\
\hline Age, years & 69 & 57 & 75 & 66 & 60 & 75 & 0.525 \\
\hline \multirow[t]{2}{*}{$\mathrm{BMI}$} & 30.8 & 25.4 & 35.8 & 28.1 & 24.0 & 32.3 & 0.091 \\
\hline & \multicolumn{3}{|l|}{$\%$} & \multicolumn{3}{|l|}{$\%$} & \\
\hline \multicolumn{7}{|l|}{ Race } & $0.885^{\mathrm{g}}$ \\
\hline Non-Hispanic white & \multicolumn{3}{|l|}{74.4} & \multicolumn{3}{|l|}{76.6} & \\
\hline Non-Hispanic black & \multicolumn{3}{|l|}{11.3} & \multicolumn{3}{|l|}{10.1} & \\
\hline Mexican-American & \multicolumn{3}{|l|}{6.6} & \multicolumn{3}{|l|}{3.6} & \\
\hline Other & \multicolumn{3}{|l|}{7.8} & \multicolumn{3}{|l|}{9.8} & \\
\hline \multicolumn{7}{|l|}{ PIR } & $0.176^{\mathrm{g}}$ \\
\hline Below poverty & \multicolumn{3}{|l|}{16.3} & \multicolumn{3}{|l|}{15.2} & \\
\hline At or above poverty & \multicolumn{3}{|l|}{81.8} & \multicolumn{3}{|l|}{73.6} & \\
\hline Missing & \multicolumn{3}{|l|}{1.9} & \multicolumn{3}{|l|}{11.2} & \\
\hline \multicolumn{7}{|l|}{ Smoking history } & $0.81^{\mathrm{g}}$ \\
\hline Current smoker & \multicolumn{3}{|l|}{20.9} & \multicolumn{3}{|l|}{18.6} & \\
\hline Former smoker & \multicolumn{3}{|l|}{23.8} & \multicolumn{3}{|l|}{21.8} & \\
\hline Never & 55.4 & & & 60.0 & & & \\
\hline HRT users & 37.3 & & & 27.2 & & & 0.532 \\
\hline Chronic diseases $^{f}$ & & & & & & & \\
\hline History of diabetes & 32.6 & & & 10.4 & & & 0.057 \\
\hline History of hypertension & 59.9 & & & 46.4 & & & 0.176 \\
\hline History of cancer & 10.4 & & & 14.8 & & & 0.506 \\
\hline History of CVD & 35.6 & & & 11.5 & & & 0.004 \\
\hline
\end{tabular}

els were associated with significantly lower odds of frailty in the multivariate analysis.

O-DMA is an intestinal bacterial metabolite of daidzein. Not all individuals harbor bacteria capable of metabolizing daidzein to O-DMA, i.e. individuals can be classified as O-DMA producers and nonproducers. About $80-95 \%$ of individuals of soy-consuming populations are O-DMA producers. Factors that may influence an individual's ability to produce O-DMA include the composition of the gut microflora, endogenous hormones, host genetics, and nonmodifiable host factors (e.g. decreasing levels with increasing age) $[20,34,35]$. O-DMA is less structurally similar to $17 \mathrm{~b}$-estradiol than its precursor compound daidzein and may exhibit different biological actions than daidzein.

Several biological actions of O-DMA have been observed in vitro, e.g. effects on cancer cells, binding to estrogen, androgen, and progesterone receptors, osteoclast formation, influencing immunological markers, and radical scavenging activity [36]. Furthermore, Low et al. [37] 
Table 3. Associations between phytoestrogen concentrations and frailty in women aged 50 years or older from NHANES 1999-2002

\begin{tabular}{llll}
\hline Phytoestrogens, $\mu \mathrm{g} / \mathrm{g}$ creatinine & $\begin{array}{l}\text { Unadjusted } \\
\text { OR }(95 \% \mathrm{CI})\end{array}$ & $\begin{array}{l}\text { Age, race/ethnicity } \\
\text { OR }(95 \% \mathrm{CI})\end{array}$ & $\begin{array}{l}\text { Multivariable adjusted } \\
\text { OR }(95 \% \mathrm{CI})\end{array}$ \\
\hline Isoflavones $^{\mathrm{b}}$ & $0.79(0.56-1.12)$ & $0.80(0.57-1.12)$ & $0.82(0.62-1.08)$ \\
Lignans $^{\mathrm{c}}$ & $0.71(0.47-1.07)$ & $0.70(0.46-1.06)$ & $0.68(0.46-1.01)$ \\
Daidzein & $0.83(0.61-1.15)$ & $0.84(0.61-1.15)$ & $0.86(0.66-1.12)$ \\
Genistein & $0.95(0.71-1.27)$ & $0.95(0.71-1.28)$ & $0.97(0.77-1.21)$ \\
O-DMA & $0.76(0.62-0.92)$ & $0.76(0.62-0.92)$ & $0.75(0.61-0.92)$ \\
Enterodiol & $0.81(0.51-1.27)$ & $0.82(0.51-1.31)$ & $0.79(0.52-1.22)$ \\
Equol among equol producers & $0.87(0.55-1.36)$ & $0.89(0.59-1.34)$ & $0.97(0.64-1.46)$ \\
Equol & $0.85(0.61-1.18)$ & $0.86(0.62-1.19)$ & $0.88(0.63-1.23)$ \\
Enterolactone & $0.81(0.61-1.07)$ & $0.81(0.61-1.07)$ & $0.80(0.60-1.07)$
\end{tabular}

Frailty was dichotomized into nonfrail and frail. ${ }^{a}$ Adjusted for age, race, PIR, HRT, smoking history, and chronic diseases (CVD, hypertension, diabetes, and cancer). ${ }^{\mathrm{b}}$ Daidzein, equol, genistein, and O-DMA. ${ }^{\mathrm{c}}$ Enterodiol and enterolactone.

stratified analyses of plasma sex hormones by presence or no presence of detectable urinary O-DMA concentrations in women aged 45-74 years who participated in EPIC-Norfolk. O-DMA-producing women had 6\% higher plasma estradiol concentrations after adjustment for potential confounding factors. Overall, O-DMA could affect the risk of frailty via various mechanisms. It is not just a marker for aging as adjustment for age did not substantially modify the effect estimate in our study.

The interpretation of the inverse association of $\mathrm{O}$ DMA with frailty in women is complex. Firstly, the crosssectional study design does not allow for establishment of the temporal sequence of events. Frailty could result from low O-DMA concentrations, but frailty is also known for an increased risk of malnutrition and this could affect the dietary intake of soy products or products containing soy. The major sources of phytoestrogens in the US diet are doughnuts, pancakes, waffles, and bread, all of which contain added soy [20]. Secondly, O-DMA may be seen as an indicator of high soy consumption, and other bioactive compounds and nutrients in soy may play a role in the prevention of frailty $[8,38-42]$. Thirdly, there is some evidence that the intestinal microflora of O-DMA producers may transform additionally polyphenolic dietary compounds, such as quercetin and kaempferol, which may reveal specific biological effects [36]. Finally, people with a high soy food intake, such as Asian people or vegetarians, may consume a more balanced diet than Americans consuming soy mainly through fortified foods, such as pancakes and doughnuts [41]. An unbalanced diet in our study population could be one of the reasons why we did not observe associations between most phytoestrogens and frailty in the present study. Furthermore, it is possible that, because this was a low-soy-consumption population, isoflavonoide and other phytoestrogen levels were too low or there was insufficient variation in the exposure to detect associations. Moreover, in the present study, the urine samples for the determination of phytoestrogen levels were spot urines, and measurements in spot urines may not be representative of the habitual nutritional intake of phytoestrogens. However, in a previous analysis of NHANES III data, urinary and serum levels of phytoestrogens showed a good correlation (daidzein $r=$ 0.72 ) [43]. In addition, given the low prevalence of frails, the power of this study is limited and further studies are warranted to illuminate the associations of phytoestrogens with frailty. On the other hand, the comparison of the women of our sample with women of the same age range not included in our sample showed that the distribution of frailty prevalence was similar.

\section{Conclusion}

Even though the observed negative association between O-DMA and frailty in women of the present study may be the result of various confounders, it may be worthwhile to intensify research on the potential preventive effects of $\mathrm{O}$ DMA. It should be taken into consideration that a low urinary concentration of O-DMA may be the result of a low intake of soy and/or of not being able to harbor the necessary intestinal microflora to produce these compounds. 


\section{Acknowledgments}

We thank all of the individuals at the NCHS of the Centers for Disease Control and Prevention who were responsible for the planning and administration of NHANES.

This work was supported by the Swiss Foundation for Nutrition Research (SFEFS; Zürich, Switzerland).

\section{Disclosure Statement}

The authors report no potential conflicts of interest.

\section{References}

1 Collerton J, Martin-Ruiz C, Davies K, Hilkens CM, Isaacs J, Kolenda C, Parker C, Dunn M, Catt M, Jagger C, von Zglinicki T, Kirkwood TB: Frailty and the role of inflammation, immunosenescence and cellular ageing in the very old: cross-sectional findings from the Newcastle 85+ Study. Mech Ageing Dev 2012; 133:456-466.

2 Fried LP, Tangen CM, Walston J, Newman AB, Hirsch C, Gottdiener J, Seeman T, Tracy R, Kop WJ, Burke G, McBurnie MA: Frailty in older adults: evidence for a phenotype. J Gerontol A Biol Sci Med Sci 2001;56:M146M156.

3 Carcaillon L, Garcia-Garcia FJ, Tresguerres JA, Gutierrez Avila G, Kireev R, RodriguezManas L: Higher levels of endogenous estradiol are associated with frailty in postmenopausal women from the Toledo Study for Healthy Aging. J Clin Endocrinol Metab 2012;97:2898-2906.

4 Michelon E, Blaum C, Semba RD, Xue QL, Ricks MO, Fried LP: Vitamin and carotenoid status in older women: associations with the frailty syndrome. J Gerontol A Biol Sci Med Sci 2006;61:600-607.

5 Paganelli R, Di Iorio A, Cherubini A, Lauretani F, Mussi C, Volpato S, Abate M, Abate G, Ferrucci L: Frailty of older age: the role of the endocrine-immune interaction. Curr Pharm Des 2006;12:3147-3159.

6 Hubbard RE, O’Mahony MS, Savva GM, Calver BL, Woodhouse KW: Inflammation and frailty measures in older people. J Cell Mol Med 2009;13:3103-3109.

7 Woods NF, LaCroix AZ, Gray SL, Aragaki A, Cochrane BB, Brunner RL, Masaki K, Murray A, Newman AB: Frailty: emergence and consequences in women aged 65 and older in the Women's Health Initiative Observational Study. J Am Geriatr Soc 2005;53:13211330.

8 Bartali B, Frongillo EA, Bandinelli S, Lauretani F, Semba RD, Fried LP, Ferrucci L: Low nutrient intake is an essential component of frailty in older persons. J Gerontol A Biol Sci Med Sci 2006;61:589-593.

9 Etman A, Burdorf A, Van der Cammen TJ, Mackenbach JP, Van Lenthe FJ: Socio-demographic determinants of worsening in frailty among community-dwelling older people in 11 European countries. J Epidemiol Community Health 2012;66:1116-1121.
10 Gill TM, Gahbauer EA, Allore HG, Han L: Transitions between frailty states among community-living older persons. Arch Intern Med 2006;166:418-423.

11 Jacobsen DE, Samson MM, Kezic S, Verhaar HJ: Postmenopausal HRT and tibolone in relation to muscle strength and body composition. Maturitas 2007;58:7-18.

12 Tanko LB, Movsesyan L, Svendsen OL, Christiansen C: The effect of hormone replacement therapy on appendicular lean tissue mass in early postmenopausal women. Menopause 2002;9:117-121.

13 Humfrey CD: Phytoestrogens and human health effects: weighing up the current evidence. Nat Toxins 1998;6:51-59.

14 Patisaul HB, Jefferson W: The pros and cons of phytoestrogens. Front Neuroendocrinol 2010;31:400-419.

15 Branca F, Lorenzetti S: Health effects of phytoestrogens. Forum Nutr 2005:100-111.

16 Setchell KD, Cole SJ: Method of defining equol-producer status and its frequency among vegetarians. J Nutr 2006;136:21882193.

17 Setchell KD, Brown NM, Lydeking-Olsen E: The clinical importance of the metabolite equol-a clue to the effectiveness of soy and its isoflavones. J Nutr 2002;132:3577-3584.

18 Frankenfeld CL: Dairy consumption is a significant correlate of urinary equol concentration in a representative sample of US adults. Am J Clin Nutr 2011;93:1109-1116.

19 Franke AA, Lai JF, Halm BM, Pagano I, Kono $\mathrm{N}$, Mack WJ, Hodis HN: Equol production changes over time in postmenopausal women. J Nutr Biochem 2012;23:573-579.

20 Valentin-Blasini L, Sadowski MA, Walden D, Caltabiano L, Needham LL, Barr DB: Urinary phytoestrogen concentrations in the US population (1999-2000). J Expo Anal Environ Epidemiol 2005;15:509-523.

21 Kuhnle GG, Dell'Aquila C, Aspinall SM, Runswick SA, Mulligan AA, Bingham SA: Phytoestrogen content of foods of animal origin: dairy products, eggs, meat, fish, and seafood. J Agric Food Chem 2008;56:1009910104.

22 Lampe JW, Atkinson C, Hullar MA: Assessing exposure to lignans and their metabolites in humans. J AOAC Int 2006;89:1174-1181.

23 Wilhelm-Leen ER, Hall YN, K Tamura, Chertow GM: Frailty and chronic kidney disease: the Third National Health and Nutrition Evaluation Survey. Am J Med 2009;122:664671.e2.

24 National Health and Nutrition Examination Survey. Laboratory Procedures Manual, 2009. Available at: http://www.cdc.gov/nchs/data/ nhanes/nhanes_09_10/lab.pdf.

25 National Center for Health Statisitcs and Centers for Disease Control and Prevention: 2001-2002 Data Documentation, Codebook, and Frequencies, 2004. Hyattsville, MD: National Center for Health Statisitcs, Centers for Disease Control and Prevention, 2012.

26 Zipf G, Chiappa M, Porter KS, et al: National Health and Nutrition Examination Survey: Plan and operations, 1999-2010. National Center for Health Statistics. Vital Health Stat 1(56), 2013.

27 Nedergaard A, Henriksen K, Karsdal MA, Christiansen C: Menopause, estrogens and frailty. Gynecol Endocrinol 2013;29:418-423.

28 Chen CY, Wu SC, Chen LJ, Lue BH: The prevalence of subjective frailty and factors associated with frailty in Taiwan. Arch Gerontol Geriatr 2010;50(suppl 1):S43-S47.

29 Santos-Eggimann B, Cuenoud P, Spagnoli J, Junod J: Prevalence of frailty in middle-aged and older community-dwelling Europeans living in 10 countries. J Gerontol A Biol Sci Med Sci 2009;64:675-681.

30 Morley JE: Diabetes, sarcopenia, and frailty. Clin Geriatr Med 2008;24:455-469, vi.

31 Klein BE, Klein R, Knudtson MD, Lee KE: Frailty, morbidity and survival. Arch Gerontol Geriatr 2005;41:141-149.

32 Strandberg TE, Sirola J, Pitkala KH, Tilvis RS, Strandberg AY, Stenholm S: Association of midlife obesity and cardiovascular risk with old age frailty: a 26-year follow-up of initially healthy men. Int J Obes (Lond) 2012;36: 1153-1157.

33 Kunisue T, Tanabe S, Isobe T, Aldous KM, Kannan K: Profiles of phytoestrogens in human urine from several Asian countries. J Agric Food Chem 2010;58:9838-9846.

34 Frankenfeld CL, Atkinson C, Thomas WK, Goode EL, Gonzalez A, Jokela T, Wahala K, Schwartz SM, Li SS, Lampe JW: Familial correlations, segregation analysis, and nongenetic correlates of soy isoflavone-metabolizing phenotypes. Exp Biol Med (Maywood) 2004; 229:902-913. 
35 Rowland I, Wiseman H, Sanders T, Adlercreutz H, Bowey E: Metabolism of oestrogens and phytoestrogens: role of the gut microflora. Biochem Soc Trans 1999;27:304-308.

36 Frankenfeld CL: O-desmethylangolensin: the importance of equol's lesser known cousin to human health. Adv Nutr 2011;2:317-324.

37 Low YL, Dunning AM, Dowsett M, Folkerd E, Doody D, Taylor J, Bhaniani A, Luben R, Khaw KT, Wareham NJ, Bingham SA: Phytoestrogen exposure is associated with circulating sex hormone levels in postmenopausal women and interact with ESR1 and NR1I2 gene variants. Cancer Epidemiol Biomarkers Prev 2007;16:1009-1016.
38 Abbatecola AM, Cherubini A, Guralnik JM, Andres Lacueva C, Ruggiero C, Maggio M, Bandinelli S, Paolisso G, Ferrucci L: Plasma polyunsaturated fatty acids and age-related physical performance decline. Rejuvenation Res 2009;12:25-32.

39 Semba RD, Bartali B, Zhou J, Blaum C, Ko CW, Fried LP: Low serum micronutrient concentrations predict frailty among older women living in the community. J Gerontol A Biol Sci Med Sci 2006;61:594-599.
40 Lee IA, Park YJ, Joh EH, Kim DH: Soyasaponin $\mathrm{Ab}$ ameliorates colitis by inhibiting the binding of lipopolysaccharide (LPS) to Tolllike receptor (TLR) 4 on macrophages. J Agric Food Chem 2011;59:13165-13172.

41 Messina M, Messina V: The role of soy in vegetarian diets. Nutrients 2010;2:855-888.

42 Willett WC: Dietary fats and coronary heart disease. J Intern Med 2012;272:13-24.

43 Valentin-Blasini L, Blount BC, Caudill SP, Needham LL: Urinary and serum concentrations of seven phytoestrogens in a human reference population subset. J Expo Anal Environ Epidemiol 2003;13:276-282. 